\title{
NON-OSCILLATION THEOREMS
}

\author{
BY \\ EINAR HILLE
}

1. Introduction. In the following we shall be concerned with the differential equation

$$
y^{\prime \prime}-F(x) y=0,
$$

where $F(x)$ is a real-valued function defined for $x>0$ and belonging to $L(\epsilon, 1 / \epsilon)$ for each $\epsilon>0$. A solution of (1.1) is a real-valued function $y(x)$, absolutely continuous together with its first derivative, which satisfies the equation for almost all $x$, in particular at all points of continuity of $F(x)$.

We shall say that the equation is non-oscillatory in $(a, \infty), a \geqq 0$, if no solution can change its sign more than once in the interval. Since the zeros of linearly independent solutions separate each other, it is sufficient that there exists a solution without zeros in the interval in order that the equation be non-oscillatory there. It is well known that (1.1) is non-oscillatory in $(0, \infty)$ if $F(x) \geqq 0$, but it may also have this property when $F(x) \leqq 0$ as is shown by the example

$$
y^{\prime \prime}+\gamma x^{-2} y=0
$$

with

$$
y(x)=C_{1} x^{\rho}+C_{2} x^{1-\rho}, \quad \rho^{2}-\rho+\gamma=0 .
$$

This equation is oscillatory for $\gamma>\frac{1}{4}$, but non-oscillatory for $\gamma \leqq \frac{1}{4}$. This example will play an important role in the following.

In this note we shall study two distinct but related problems.

I. When does equation (1.1) have a solution which tends to a limit $\neq 0, \infty$ when $x \rightarrow \infty$ ?

II. If $F(x)=-f(x)$ where $f(x)$ is non-negative, when is equation (1.1) nonoscillatory in $(a, \infty)$ for a sufficiently large?

It should be noted that if $F(x)=-f(x) \leqq 0$, then a solution of Problem I is also a solution of Problem II but not vice versa. The impetus to the present investigation was given by a recent paper by Aurel Wintner ([10] in the appended bibliography) in which these problems were mentioned. Wintner proved (loc. cit. pp. 96-97) that $f(x) \in L(1, \infty)$ is a necessary condition for both I and II; in studying problem I he restricted himself to the case in which $F(x)=f(x) \geqq 0$. Example (1.2) shows, however, that the condition is not sufficient in either case.

Problem I, though not trivial lies fairly close to the surface. Assuming

Presented to the Society, September 5, 1947; received by the editors June 3, 1947. 
$F(x)$ to be of constant sign at least for large values of $x$, it is not difficult to strengthen Wintner's necessary condition to $x F(x) \in L(1, \infty)$ and it is an easy matter to show that this condition is also sufficient. Actually a special case of Problem I was attacked by the present writer in 1924 [4, p. 491] in connection with the equation

$$
w^{\prime \prime}+\left[a^{2}-F(z)\right] w=0
$$

in which the perturbation term $F(z)$ is supposed to be holomorphic in a right half-plane and $|F(x+i y)| \leqq M(x)$ where $M(x)$ is monotone decreasing and satisfies a suitable condition of integrability $\left({ }^{1}\right)$. The case which has a bearing on Problem I is that in which $a=0$; assuming $x M(x) \in L(0, \infty)$, I could prove the existence of a solution $w_{1}(z)$ of (1.4) such that $w_{1}(z)=1+o(1)$ for large $|z|$. This is the exceptional or sub-dominant solution; assuming the stronger condition $x^{2} M(x) \in L(0, \infty)$, I could isolate a dominant solution $w_{2}(z)=z$ $+o(1)$ for large $|z|$. The proof is based upon the method of successive approximations and applies just as well if $F(\cdot)$ is defined only for real values of the variable. The assumption that $M(x)$ is monotone turns out to be superflous and it is an easy matter to show that $x F(x) \in L(1, \infty)$ suffices for the existence of the exceptional solution. The details of the discussion are given in $\$ 2$ below.

Problem II on the other hand is much more refractory. Wintner's necessary condition, $f(x) \in L(1, \infty)$, can be strengthened to, for instance, $x^{\sigma} f(x)$ $\in L(1, \infty)$ for each $\sigma<1$, but not for $\sigma=1$. Example (1.2) shows that even this stronger condition is not sufficient. More significant is the observation that the function

$$
g(x)=x \int_{x}^{\infty} f(t) d t
$$

stays bounded for large $x$ if (1.1) is non-oscillatory. More precisely, if the inferior and superior limits of $g(x)$ for $x \rightarrow \infty$ are denoted by $g_{*}$ and $g^{*}$ respectively, then $g_{*} \leqq \frac{1}{4}, g^{*} \leqq 1$ are necessary and $g^{*}<\frac{1}{4}$ sufficient conditions in order that (1.1) be non-oscillatory for large $x$. Here all inequalities are sharp. The proof of this result is given in $\$ 3$, the necessary counter examples are constructed in $\$ 4$.

Example (1.2) was taken as the point of departure for a study of Problem II by A. Kneser [5, pp. 414-418] in $1892\left({ }^{2}\right)$. He proved that if

(1) There is a considerable literature on this and related problems. Of recent papers, reference should be made to R. Bellman [1] and N. Levinson [6]. The idea of reducing the study of (1.4) to a singular integral equation which is solved by the method of successive approximations goes back to E. Cotton [2]. The case $a=0$, in which at most one solution can remain bounded, seems to have been disregarded in the literature.

(2) I am indebted to a referee for calling my attention to the fact that Problem II goes back to Kneser. A further search of the literature led to Riemann-Weber [7] where the discus- 
$\lim _{x \rightarrow \infty} x^{2} f(x)=\gamma$, then (1.1) is oscillatory for $\gamma>\frac{1}{4}$ and non-oscillatory for $\gamma<\frac{1}{4}$. If $\gamma=\frac{1}{4}$ no conclusion can be drawn unless $x^{2} f(x)<\frac{1}{4}$ for all large $x$. In $\$ 5$ we shall exhibit an infinite sequence of differential equations which lead to successive refinements of Kneser's criterion of a nature similar to the logarithmic scale in the theory of convergence of infinite series. In this discussion one may replace limits by inferior and superior limits, but no conclusion can be drawn if $\frac{1}{4}$ lies in the interval of indetermination of the critical ratio in question.

In the study of Problem II the constant $\frac{1}{4}$ plays a peculiar role. It is the value of $\gamma$ for which the quadratic equation $\rho^{2}-\rho+\gamma=0$ has equal roots. This equation was noted above in (1.3) where it occurred as the indicial equation in the sense of Fuchs of equation (1.2). It plays a similar role for the other equations of the logarithmic scale, but it also enters the argument in a different way. The main tool in our study of Problem II is the associated Riccati equation

$$
v^{\prime}+v^{2}+f(x)=0, \quad v=\frac{y^{\prime}}{y},
$$

and the corresponding singular integral equation which in the non-oscillatory case can be written in the form

$$
u(x)=x \int_{x}^{\infty} u^{2}(t) \frac{d t}{t^{2}}+g(x), \quad u(x)=x v(x),
$$

with $g(x)$ defined by (1.5). This equation shows that the behavior of $g(x)$ for large $x$ is decisive for that of $u(x)$; in particular, lim $u(x)=\rho$ can exist if and only if $\lim g(x)=\gamma$ exists in which case $\rho^{2}-\rho+\gamma=0$ and $\gamma \leqq \frac{1}{4}$. It is perhaps of some interest to observe that here is a linear problem which, apparently, is best studied by nonlinear methods.

Finally, in $\$ 6$ we give a partial extension of Problem II to the complex plane which also is based upon equation (1.7).

2. Problem I. Let $F(x)$ be a real-valued function, defined for $x>0$ and belonging to $L(\epsilon, 1 / \epsilon)$ for each $\epsilon>0$. We shall say that the differential eguation

sion of oscillation theorems (pp. 53-72, 5th ed., omitted in the 7th Frank-v. Mises ed.) is based on Kneser. The case in which $f(x) \rightarrow 0$ is considered on pp. 60-62, but here the discussion goes beyond Kneser. If $\gamma=\frac{1}{4}$, Weber puts $y=x^{1 / 2} \eta, \xi=\log x$; this gives a new differential equation to which Kneser's criterion may be applied. The resulting theorem is equivalent to our Theorem 10 below. Weber also indicates how the iteration of this process leads to an infinite chain of conditions. The existence of this passage in Riemann-Weber had escaped my memory at the time of deriving Theorems 10 and 11 . Though these theorems are not essentially new, I have not suppressed them from the text since they provide necessary background for the rest of the discussion. For further literature relating to Problem II, see also W. B. Fite [3, p. 343] and A. Wiman [9, pp. 4-5]. Wiman has added much to our knowledge of the fine structure of the solutions, but for the case of Problem II he does not go beyond Kneser, nor does he claim to do so. [Revised September 9, 1947.] 


$$
y^{\prime \prime}-F(x) y=0
$$

has property $\mathrm{I}$ if it admits of a solution $y_{1}(x)$ such that

$$
\lim _{x \rightarrow \infty} y_{1}(x)=1 \text {. }
$$

LeMma 1. If (2.1) has property $\mathrm{I}$, then its general solution is of the form

$$
y(x)=C_{1}\left[1+\epsilon_{1}(x)\right]+C_{2} x\left[1+\epsilon_{2}(x)\right],
$$

where $C_{1}$ and $C_{2}$ are arbitrary constants and $\lim _{x \rightarrow \infty} \epsilon_{k}(x)=0$.

Proof. By assumption there exists a solution $y_{1}(x)=1+\epsilon_{1}(x)$. Let $a$ be so large that $\left|\epsilon_{1}(x)\right|<1$ for $x \geqq a$. Then

$$
y_{2}(x)=y_{1}(x) \int_{a}^{x} \frac{d t}{\left[y_{1}(t)\right]^{2}}=x\left[1+\epsilon_{2}(x)\right]
$$

exists and is also a solution of (2.1) as is well known and easily verified.

Lemma 2. If (2.1) has property $\mathrm{I}$, then (2.1) is non-oscillatory for large $x$. Moreover, if $F(x)$ keeps a constant sign in $(c, \infty), c>0$, then every solution $y(x)$ is ultimately monotone and $y^{\prime}(x)$ tends to a finite limit when $x \rightarrow \infty$. Further, $y(x)$ is ultimately convex or concave towards the $x$-axis according as $F(x) \geqq 0$ or $F(x) \leqq 0$.

Proof. If $a$ is chosen as above, then $y_{1}(x) \neq 0$ for $x \geqq a$ and no solution can have more than one zero in $(a, \infty)$. Suppose that $F(x)$ keeps a constant sign for $x \geqq c$, say $F(x) \geqq 0$, and that a solution $y(x)$ is positive for $c \leqq b \leqq x$. If $b \leqq x_{1}<x_{2}<\infty$, then

$$
y^{\prime}\left(x_{2}\right)-y^{\prime}\left(x_{1}\right)=\int_{x_{1}}^{x_{2}} F(t) y(t) d t
$$

is never negative. Hence $y^{\prime}(x)$ is never decreasing for $x \geqq b$ and tends to a limit when $x \rightarrow \infty$. This limit must be finite because $y(x)<K x$ for large $x$ by (2.3). The convexity properties are obvious.

THEOREM 1. If $F(x)$ keeps a constant sign for large $x$ and if (2.1) has property $\mathrm{I}$, then $x F(x) \in L(1, \infty)$.

Proof. We take $C_{1}=0, C_{2}=1$ in (2.3) and substitute in (2.4). Since the left side tends to a finite limit when $x_{2} \rightarrow \infty$, so does the right, that is $F(x) y(x)$ $\in L(a, \infty)$ because $F(x) y(x)$ ultimately keeps a constant sign. But $y(x)>\frac{1}{2} x$ for large $x$ and $F(x) \in L(1, a)$. Hence $x F(x) \in L(1, \infty)$.

Theorem 2. If $x F(x) \in L(1, \infty)$, then (2.1) has property I. Moreover

$$
\left|y_{1}(x)-1\right| \leqq \exp [G(x)]-1, \quad G(x)=\int_{x}^{\infty} t|F(t)| d t .
$$


Proof. Consider the integral equation

$$
Y(x)=1+\int_{x}^{\infty}(t-x) F(t) Y(t) d t
$$

and define

$$
Y_{0}(x)=1, \quad Y_{k}(x)=1+\int_{x}^{\infty}(t-x) F(t) Y_{k-1}(t) d t .
$$

We have obviously

$$
\left|Y_{1}(x)-Y_{0}(x)\right| \leqq \int_{x}^{\infty}(t-x)|F(t)| d t \leqq G(x) .
$$

Using the fact that $G^{\prime}(x)=-x|F(x)|$ for almost all $x$, it is a simple matter to verify the estimate

$$
\left|Y_{k}(x)-Y_{k-1}(x)\right| \leqq \frac{1}{k !}[G(x)]^{k}, \quad k=1,2,3, \cdots .
$$

From this it follows that $Y_{k}(x)$ converges to a limit $Y(x)$ uniformly for $x \geqq \epsilon>0$, and $Y(x)$ satisfies (2.5) and (2.6). Differentiating (2.6) twice with respect to $x$, we see that $Y(x)$ satisfies $(2.1)$ for almost all positive $x$. Thus $Y(x)$ is the desired solution $y_{1}(x)$ and $(2.1)$ has property $I\left({ }^{3}\right)$. This completes the proof.

COROLlaRY. If $F(x)$ keeps a constant sign for large $x$, then $x F(x) \in L(1, \infty)$ is necessary and sufficient in order that equation (2.1) have property $I$.

This is a solution of Problem I. The condition remains sufficient if $F(x)$ is allowed to be complex-valued, but the necessity is lost already when we drop the assumption that $F(x)$ keeps a constant sign for large $x$. This is shown by the following simple example. The function

$$
y(x)=1-\frac{\sin x}{x}
$$

satisfies a differential equation of type (2.1) with

$$
F(x)=\frac{\left(x^{2}-2\right) \sin x+2 x \cos x}{x^{2}(x-\sin x)}
$$

as is shown by computing $y^{\prime \prime} / y$. The corresponding equation has property I

(3) Differentiation in (2.6) shows that $y_{1}^{\prime}(x) \rightarrow 0$ when $x \rightarrow \infty$ and more precise information is readily obtained from the equation if desired. Similarly, in the case of the dominant solution $y_{2}(x)$ of Theorem 3 we have $y_{2}^{\prime}(x) \rightarrow 1$. It should be added that the estimates (2.5) and (2.11) differ from the corresponding estimates on p. 491 of [4]. The difference is not essential, but may conceivably be due to an error of calculation in the older estimates. 
but neither $F(x)$ nor $x F(x)$ belongs to $L(1, \infty)$.

For large values of $x$ the inequality (2.5) may be replaced by the more favorable estimate

$$
\begin{aligned}
\left|y_{1}(x)-1\right| & <F_{2}(x)\left[1-F_{2}(x)\right]^{-1}, \\
F_{2}(x) & =\int_{x}^{\infty}(t-x)|F(t)| d t,
\end{aligned}
$$

which is valid for $F_{2}(x)<1$. This follows from the estimate $\left|Y_{k}(x)-Y_{k-1}(x)\right|$ $<\left[F_{2}(x)\right]^{k}$ which is easily verified.

We shall say that the differential equation (2.1) has property $\mathrm{I}^{*}$ if there exists a solution $y_{2}(x)$ such that

$$
\lim _{x \rightarrow \infty}\left[y_{2}(x)-x\right]=0
$$

Theorem 3. Property I* implies property I but not vice versa. The equation has property $\mathrm{I}^{*}$ if $x^{2} F(x) \in L(1, \infty)$ and, if $F(x)$ keeps a constant sign for large $x$, then the condition is necessary as well as sufficient.

Proof. If (2.1) has property $I^{*}$ then (as in the proof of Lemma 1)

$$
y_{1}(x)=y_{2}(x) \int_{x}^{\infty}\left[y_{2}(t)\right]^{-2} d t=1+o\left(\frac{1}{x}\right)
$$

is a solution of (2.1), so that (2.1) has property I. In particular, if $F(x)$ keeps a constant sign for large $x$, we see that $x F(x) \in L(1, \infty)$. This condition also implies property $I$, but, as we shall see below, the stronger condition $x^{2} F(x)$ $\in L(1, \infty)$ is required for property $I^{*}$ when $F(x)$ keeps a constant sign.

In order to prove that $x^{2} F(x) \in L(1, \infty)$ is a sufficient condition for property I*, we proceed as in the proof of Theorem 2, replacing (2.6) by the new singular integral equation

$$
Z(x)=x+\int_{x}^{\infty}(t-x) F(t) Z(t) d t
$$

The details can be left to the reader; we note, however, the resulting estimate

$$
\left|y_{2}(x)-x\right|<\exp [H(x)]-1, \quad H(x)=\int_{x}^{\infty} t^{2}|F(t)| d t .
$$

The proof of the necessity is more interesting. Suppose that $F(x)$ keeps a constant sign for large $x$ and that (2.1) has property I*. As observed above, it has also property I and if $y_{1}(x)$ denotes the solution defined by (2.9) we have conversely

$$
y_{2}(x)=y_{1}(x) \int_{a}^{x}\left[y_{1}(t)\right]^{-2} d t+C y_{1}(x) .
$$


Here we choose $a$ so large that (i) sgn $F(x)$ is constant and (ii) $\left|y_{1}(x)-1\right|<\frac{1}{5}$ for $x>a$. After $a$ has been chosen, $C$ is uniquely determined but its actual value is of no importance for the following. We set

$$
y_{1}(x)=1+\gamma(x)
$$

and expand $[1+\gamma(t)]^{-2}$ in powers of $\gamma(t)$, using two terms and the exact form of the remainder. The result may be written

$$
\begin{aligned}
& y_{2}(x)-(x-a)-C[1+\gamma(x)]=\left[\gamma(x)(x-a)-\int_{a}^{x} \gamma(t) d t\right] \\
&-[1+2 \gamma(x)] \int_{a}^{x} \gamma(t) d t+[1+\gamma(x)] \int_{a}^{x} \frac{3 \gamma^{2}(t)+2 \gamma^{3}(t)}{[1+\gamma(t)]^{2}} d t .
\end{aligned}
$$

By assumption, the left side tends to the limit $a-C$ when $x \rightarrow \infty$ so the right member must also tend to the same limit. We shall show that this implies that $\int_{a}^{x} \gamma(t) d t$ tends to a finite limit.

By (2.6), which admits of $y_{1}(x)$ as its unique solution, we have

$$
\gamma(x)=\int_{x}^{\infty}(t-x) F(t) y_{1}(t) d t, \quad \gamma^{\prime}(x)=-\int_{x}^{\infty} F(t) y_{1}(t) d t
$$

so that $\operatorname{sgn} \gamma(x)=\operatorname{sgn} F(x)$, sgn $\gamma^{\prime}(x)=-\operatorname{sgn} F(x)$ for $x>a$ and $|\gamma(x)|$ is monotone decreasing to zero.

There are two cases to consider.

(i) $F(x) \geqq 0$ for $x>a$. In this case the first term in the right member of (2.12) is negative while the third term does not exceed 0.567 times $\int_{a}^{x} \gamma(t) d t$. It follows that the latter integral must tend to a finite limit when $x \rightarrow \infty$ and this implies that $\gamma(x) \in L(1, \infty)$.

(ii) $F(x) \leqq 0$ for $x>a$. In this case all three terms in the right member of (2.12) are positive and the same conclusion holds.

But

$$
\int_{a}^{\infty}|\gamma(t)| d t=\int_{a}^{\infty} \int_{t}^{\infty}(s-t)|F(s)| y_{1}(s) d s d t
$$

exists if and only if

$$
\int_{a}^{\infty} \int_{t}^{\infty}(s-t)|F(s)| d s d t=\frac{1}{2} \int_{a}^{\infty}(s-a)^{2}|F(s)| d s
$$

exists, that is, if and only if $x^{2} F(x) \in L(1, \infty)$. This completes the proof.

If $(2.1)$ has property $I^{*}$ then

$$
\lim _{x \rightarrow \infty}\left[C_{1} y_{1}(x)+C_{2} y_{2}(x)-C_{1}-C_{2} x\right]=0 .
$$


Expressed in geometrical language, this leads to the following.

COROLlaRy. There is a one-to-one correspondence between the integral curves of (2.1) and the non-vertical straight lines. Every integral curve has a unique slanting asymptote, distinct integral curves having distinct asymptotes, and every slanting straight line is the asymptote of a unique integral curve.

The theory of Bessel functions provides illustrations of the results of the present section. If $F(x)=x^{-2-\alpha}$, then the corresponding equation has property I for $\alpha>0$ and property $\mathrm{I}^{*}$ for $\alpha>1$. The corresponding subdominant solution $y_{1}(x)$ is a constant multiple of

$$
x^{1 / 2} J_{1 / \alpha}\left(\frac{2 i}{\alpha} x^{-\alpha / 2}\right)
$$

a result which goes back to Euler. Similarly if $F(x)=-e^{-2 x}$ we have $y_{1}(x)$ $=J_{0}\left(e^{-x}\right)$. Dominant solutions involve functions of the second kind. See G. N. Watson [8, pp. 96 and 99].

3. Problem II. Let $f(x)$ be a non-negative function defined for $x>0$ and belonging to $L(\epsilon, 1 / \epsilon)$ for each $\epsilon>0$. We shall say that the differential equation

$$
y^{\prime \prime}+f(x) y=0
$$

has the property II if it is non-oscillatory for large $x$.

LEMMA 3. If (3.1) has property II and if $y(x)$ is a solution which is positive for $x \geqq a$, then $y(x)$ is monotone increasing and concave downwards for $x>a$. Further $y^{\prime}(x)$ is positive and monotone decreasing towards a limit $\geqq 0$.

Proof. From (2.4) we get

$$
y^{\prime}\left(x_{2}\right)-y^{\prime}\left(x_{1}\right)=-\int_{x_{1}}^{x_{2}} f(t) y(t) d t
$$

which is non-positive for $a<x_{1}<x_{2}$. It follows that $y^{\prime}(x)$ is never increasing for $a<x$ and $y(x)$ is concave downwards. Since the graph of $y=y(x)$ lies below the curve tangent and does not intersect the $x$-axis for $x>a$, we must have $y^{\prime}(x)>0$.

Formula (3.2) shows that $y(x) f(x) \in L(1, \infty)$, but all that can be concluded from Lemma 3 concerning the growth of $y(x)$ is that $|y(x)| \leqq K x$ for large $x$. If the converse inequality should hold for a particular $y(x)$, then $x f(x) \in L(1, \infty)$ as we know from the preceding discussion, but this property is not necessary for the equation to have property II. For a further study of this matter we resort to the associated Riccati equation

$$
v^{\prime}+v^{2}+f(x)=0, \quad v=y^{\prime} / y .
$$

LEMMA 4. If (3.1) has property II and if $y(x) \neq 0$ for $x \geqq a$, then 


$$
0<(x+c) v(x) \leqq 1, \quad c=-a+1 / v(a), \quad v(x)=y^{\prime}(x) / y(x) .
$$

Proof. For $x \geqq a$ the function $v(x)$ is absolutely continuous and satisfies (3.3) for almost all $x$. By Lemma 3,v(x)>0 for $x \geqq a$ and (3.3) shows that it is a never increasing function of $x$. Moreover, $v^{\prime}+v^{2} \leqq 0$ whence it follows that

$$
\frac{d}{d x}\left[-\frac{1}{v}+x\right] \leqq 0
$$

and

$$
-1 / v(x)+x \leqq-1 / v(a)+a, \quad a \leqq x
$$

which is (3.4).

Corollary. We have

$$
0 \leqq \limsup _{x \rightarrow \infty} x v(x) \leqq 1
$$

Next we proceed to strengthen Wintner's necessary condition.

LEMMA 5. If $\mu(x)$ is a positive never decreasing function, if $\mu(x) x^{-2} \in L(1, \infty)$, and if (3.1) has property II, then $\mu(x) f(x) \in L(1, \infty)$.

Proof. We choose $a$ as in Lemma 4, $y(x)$ being a given solution of (3.1). We then multiply (3.3) by $\mu(x)$ and integrate between the limits $a$ and $b$, obtaining after an integration by parts,

$$
\begin{aligned}
v(b) \mu(b)-v(a) \mu(a) & -\int_{a}^{b} v(t) d \mu(t) \\
& +\int_{a}^{b} \mu(t) v^{2}(t) d t+\int_{a}^{b} \mu(t) f(t) d t=0
\end{aligned}
$$

The assumptions on $\mu(x)$ together with (3.4) show that the second integral in this formula tends to a finite limit when $b \rightarrow \infty$ and for the same reason $\mu(b) v(b) \rightarrow 0$. Further

$$
\begin{aligned}
\int_{a}^{b} v(t) d \mu(t) & \leqq \int_{a}^{b} \frac{d \mu(t)}{t+c}=\frac{\mu(b)}{b+c}-\frac{\mu(a)}{a+c}+\int_{a}^{b} \frac{\mu(t)}{(t+c)^{2}} d t \\
& \rightarrow-\frac{\mu(a)}{a+c}+\int_{a}^{\infty} \frac{\mu(t)}{(t+c)^{2}} d t
\end{aligned}
$$

It follows that the last integral in (3.6) also tends to a finite limit and the theorem is proved.

Admissible choices of $\mu(x)$ are given by the functions

$$
x^{\sigma}, \quad \sigma<1 ; \quad x(\log x)^{-1-\alpha} ; \quad x(\log x)^{-1}(\log \log x)^{-1-\alpha}, \quad \alpha>0 .
$$


In particular we may take $\mu(x)=1$; this leads to the integral equation

$$
v(x)=\int_{x}^{\infty} v^{2}(t) d t+\int_{x}^{\infty} f(t) d t
$$

which is basic for the following discussion.

THEOREM 4. Equation (3.1) has property II if and only if the integral equation (3.7) has a solution for sufficiently large values of $x$.

Proof. The necessity has already been proved. Suppose that there is a finite $a$ such that (3.7) has a solution for $x \geqq a$. From the form of the equation it follows that $v^{2}(x) \in L(a, \infty)$ and $v(x)$ is a positive, monotone decreasing, absolutely continuous function. Differentiation with respect to $x$ shows that $v(x)$ satisfies (3.3) for almost all $x$. Hence if we put

$$
y(x)=\exp \left[\int_{a}^{x} v(t) d t\right]
$$

then $y(x)$ satisfies (3.1) for almost all $x$ and for $x \geqq a$ we have $y(x) \geqq 1$ so that (3.1) has property II.

For the discussion of (3.7) let us introduce the following notation

$$
u(x)=x v(x), \quad g(x)=x \int_{x}^{\infty} f(t) d t
$$

in terms of which (3.7) becomes

$$
u(x)=x \int_{x}^{\infty} u^{2}(t) \frac{d t}{t^{2}}+g(x) .
$$

We also write

$$
\lim \sup _{\inf } u(x)=\left\{\begin{array}{l}
u^{*} \\
u_{*}
\end{array}, \quad \lim \sup _{\inf } g(x)=\left\{\begin{array}{l}
g^{*} \\
g_{*}
\end{array} .\right.\right.
$$

THEOREM 5. If (3.1) has property II, then $g_{*} \leqq \frac{1}{4}$ and $g^{*} \leqq 1$. Both estimates are the best possible of their kind.

Proof. Since (3.1) is assumed to have properly II, equation (3.9) has solutions for large values of $x$ and by the corollary of Lemma 4 we have $0 \leqq u_{*}$ $\leqq u^{*} \leqq 1$. The first term on the right of (3.9) is positive so that

$$
g_{*} \leqq u_{*}, \quad g^{*} \leqq u^{*},
$$

and the second inequality gives $g^{*} \leqq 1$ as asserted. Since

$$
\left(u_{*}\right)^{2}-\epsilon \leqq x \int_{x}^{\infty} u^{2}(t) \frac{d t}{t^{2}} \leqq\left(u^{*}\right)^{2}+\epsilon
$$


for $x \geqq x_{\epsilon},(3.9)$ also shows that

$$
u_{*} \geqq\left(u_{*}\right)^{2}+g_{*}, \quad u^{*} \leqq\left(u^{*}\right)^{2}+g^{*} .
$$

The first of these inequalities requires that $g_{*} \leqq \frac{1}{4}$; it then gives

$$
\frac{1}{2}-\left(\frac{1}{4}-g_{*}\right)^{1 / 2} \leqq u_{*} \leqq \frac{1}{2}+\left(\frac{1}{4}-g_{*}\right)^{1 / 2} .
$$

The second inequality under (3.12) is true for all $u^{*}$ if $g^{*} \geqq \frac{1}{4}$. It imposes a restriction on $u^{*}$ if $g^{*}<\frac{1}{4}$ in which case it shows that either

$$
u^{*} \leqq \frac{1}{2}-\left(\frac{1}{4}-g^{*}\right)^{1 / 2} \text { or } \frac{1}{2}+\left(\frac{1}{4}-g^{*}\right)^{1 / 2} \leqq u^{*} .
$$

This completes the proof of the inequalities. Example (1.2) with $\gamma=\frac{1}{4}$ shows that $g_{*}$ may equal $\frac{1}{4}$ when the equation has property II. An example with $g^{*}=1$ will be constructed in $\$ 4$.

THEOREM 6. If (3.1) has property II and if for a particular solution $y_{1}(x)$ of (3.1) the corresponding function $u_{1}(x)$ tends to a limit $\rho$ when $x \rightarrow \infty$, then $\lim _{x \rightarrow \infty} g(x)=\gamma$ exists and $\rho^{2}-\rho+\gamma=0$. Further $\lim _{x \rightarrow \infty} u(x)$ exists for every solution of (3.1) and is either $\rho$ or $1-\rho$. There exists a solution for which the limit equals $1-\rho$.

Proof. The first assertion is an immediate consequence of equation (3.9) and the latter also shows that if $\lim _{x \rightarrow \infty} u(x)$ exists for any other solution of (3.1), then the limit is either $\rho$ or $1-\rho$. But to prove the existence of the limit requires a more elaborate argument than one would expect at first sight. The crux of the proof lies in showing the existence of a solution $y_{2}(x)$ with lim $u_{2}(x)$ $=1-\rho$. There are two distinct cases according as $\left[y_{1}(x)\right]^{-2} \in L(a, \infty)$ or not. The first case is present, for a suitable choice of $a$, if $\frac{1}{2}<\rho \leqq 1$, the second if $0 \leqq \rho<\frac{1}{2}$, while $\rho=\frac{1}{2}$ may belong to either case. We shall carry through the argument in the first case. We form

$$
y_{2}(x)=y_{1}(x) \int_{x}^{\infty}\left[y_{1}(t)\right]^{-2} d t
$$

which exists and is a solution of (3.1) under the present assumptions provided $x>a$. Logarithmic differentiation or use of the Wronskian yields

$$
u_{2}(x)=u_{1}(x)-x / P(x), \quad P(x)=y_{1}(x) y_{2}(x) .
$$

But an integration by parts gives

$$
\begin{aligned}
P(x) & =\left[y_{1}(x)\right]^{2} \int_{x}^{\infty}\left[y_{1}(t)\right]^{-2} d t \\
& =-x+2\left[y_{1}(x)\right]^{2} \int_{x}^{\infty} u_{1}(t)\left[y_{1}(t)\right]^{-2} d t \\
& =-x+2 \rho P(x)+o[P(x)]
\end{aligned}
$$


since $u_{1}(t) \rightarrow \rho$ when $t \rightarrow \infty$. Hence

$$
x / P(x)=2 \rho-1+o(1), \quad \rho \geqq 1 / 2,
$$

and $\lim u_{2}(x)=1-\rho$. If we set $y_{2}(x) / y_{1}(x)=R(x)$, then $\lim _{x \rightarrow \infty} R(x)=0$. Hence, if $y(x)=C_{1} y_{1}(x)+C_{2} y_{2}(x)$ where $C_{1} \neq 0$, then

$$
u(x)=\frac{C_{1} u_{1}(x)+C_{2} u_{2}(x) R(x)}{C_{1}+C_{2} R(x)} \rightarrow \rho .
$$

Thus the limit of $u(x)$ always exists and equals $\rho$ unless $C_{1}=0$ in which case it becomes $1-\rho$. We note that if $\rho=\frac{1}{2}$ so that the two limits coincide, then $y_{1}(x)$ and $y_{2}(x)$ are still linearly independent solutions of (3.1).

If $\left[y_{1}(x)\right]^{-2}$ is not in $L(a, \infty)$ for any $a$, we modify the definition of $y_{2}(x)$, replacing the integral from $x$ to $\infty$ by one from $a$ to $x$ having the same integrand. The proof then goes through as in the first case.

The theorem shows that a necessary condition for the existence of $\lim u(x)$ for any solution $y(x)$ of (3.1) is the existence of $\lim g(x)$ which is then necessarily $\leqq \frac{1}{4}$. We shall prove in Theorem 8 below that, conversely, the existence of $\lim g(x)$ implies that of $\lim u(x)$ provided the first limit is $<\frac{1}{4}$.

We shall now prove a comparison theorem which leads to sufficient conditions for property II.

THEOREM 7. Given the differential equations

$$
\begin{aligned}
Y^{\prime \prime}+F(x) Y=0, & G(x)=x \int_{x}^{\infty} F(t) d t, \\
y^{\prime \prime}+f(x) y=0, & g(x)=x \int_{x}^{\infty} f(t) d t .
\end{aligned}
$$

If the first equation has property II and if $G(x) \geqq g(x)$ for $x \geqq a$, then the second equation also has property II.

Proof. By Theorem 4 the integral equation

$$
U(x)=x \int_{x}^{\infty} U^{2}(t) \frac{d t}{t^{2}}+G(x)
$$

has a solution $U(x)$, defined for $x \geqq b$ say. We now consider equation (3.9) for $x \geqq c=\max (a, b)$ and define successive approximations by

$$
u_{0}(x)=U(x), \quad u_{n}(x)=x \int_{x}^{\infty} u_{n-1}^{2}(t) \frac{d t}{t^{2}}+g(x) .
$$

Here

$$
u_{1}(x)=x \int_{x}^{\infty} U^{2}(t) \frac{d t}{t^{2}}+g(x) \leqq x \int_{x}^{\infty} U^{2}(t) \frac{d t}{t^{2}}+G(x)=U(x)=u_{0}(x)
$$


Since

$$
u_{n}(x)-u_{n-1}(x)=x \int_{x}^{\infty}\left[u_{n-1}^{2}(t)-u_{n-2}^{2}(t)\right] \frac{d t}{t^{2}},
$$

we see that $u_{n-1}(x) \geqq u_{n}(x) \geqq g(x)$ for all $x$ and all $n$. Hence lim $u_{n}(x)=u(x)$ exists and satisfies (3.9). Using Theorem 4 once more we see that equation $(3.16)=(3.1)$ has property II as asserted.

COROLlARY 1. Equation (3.1) has property II if $g(x) \leqq \frac{1}{4}$ for $x \geqq a$. This holds, in particular, if $g^{*}<\frac{1}{4}$.

For $G(x) \equiv \frac{1}{4}$ corresponds to $F(x)=\frac{1}{4} x^{-2}$ and $Y(x)=x^{1 / 2}\left(C_{1}+C_{2} \log x\right)$ so that the corresponding equation (3.15) has property II. Another sufficient condition will be proved in $\$ 5$ (Theorem 12).

COROLlaRy 2. If $U(x)$ is any solution of (3.17) defined for $x \geqq c$, and if $G(x) \geqq g(x)$ for $x \geqq c$, then there exists a solution $u(x)$ of (3.9) with $u(x) \leqq U(x)$ for $x \geqq(c)$.

This was proved above.

THEOREM 8. If $\lim _{x \rightarrow \infty} g(x)=\gamma$ exists and if $\gamma<\frac{1}{4}$, then $\lim _{x \rightarrow \infty} u(x)$ $=\lim _{x \rightarrow \infty} x y^{\prime}(x) / y(x)$ exists for every solution $y(x)$ of $(3.1), y(x) \not \equiv 0$.

Proof. By Theorem 6 it is sufficient to prove the existence of a single solution $u_{1}(x)$ of (3.9) such that $\lim u_{1}(x)$ exists. We shall base the proof on Corollary 2. Given an $\epsilon>0$, we can find an $a$ such that $\gamma-\epsilon \leqq g(x) \leqq \gamma+\epsilon$ for $x \geqq a$. If $\gamma=0$ we may replace $\gamma-\epsilon$ by 0 ; we may also suppose that $\gamma+\epsilon \leqq \frac{1}{4}$. Consider the quadratic equation $u^{2}-u+g=0$ with $g=\gamma-\epsilon$ or $\gamma+\epsilon$ and denote the smaller of its roots by $\rho$ and $\sigma$ respectively so that $\rho<\sigma \leqq \frac{1}{2}$. Together with (3.9) we consider the two auxiliary equations obtained by replacing $g(x)$ by $\boldsymbol{\gamma} \boldsymbol{\epsilon}$ and $\boldsymbol{\gamma}+\boldsymbol{\epsilon}$ respectively, that is,

$$
\begin{aligned}
& L(x)=x \int_{x}^{\infty} L^{2}(t) \frac{d t}{t^{2}}+\gamma-\epsilon, \\
& U(x)=x \int_{x}^{\infty} U^{2}(t) \frac{d t}{t^{2}}+\gamma+\epsilon .
\end{aligned}
$$

The first equation has two constant solutions $\rho$ and $1-\rho$; every solution tends to a limit when $x \rightarrow \infty$ and the limit is $1-\rho$ unless $L(x) \equiv \rho$. The same description holds for the second equation if we replace $\rho$ by $\sigma$.

By Corollary 2 there is a solution of (3.9), $u(x)$ say, such that $u(x)$ $\leqq U(x) \equiv \sigma$, and there is a solution $L(x)$ of (3.18) with $L(x) \leqq u(x)$. But $L(x)$ tends to a limit when $x \rightarrow \infty$; the limit being $<\frac{1}{2}$, it must be $\rho$. Hence $L(x) \equiv \rho$ and $\rho \leqq u(x) \leqq \sigma$ when $x \geqq a$. Since $\sigma-\rho \leqq 2 \epsilon^{1 / 2}$ we see that $\lim _{x \rightarrow \infty} u(x)$ must exist and the theorem is proved. 
Another application of the ideas underlying Theorem 7 will be given in $\S 6$.

4. Counter examples. In order to show that the results proved in $\S 3$ are not capable of essential improvement we shall exhibit some counter examples. $g^{*}=1$.

A. There exists a differential equation having property II for which $g_{*}=0$,

Construction. If $y=y(x)$ is a positive, monotone increasing function whose graph is concave downwards, and if $y^{\prime}(x)$ is continuous, then $y(x)$ satisfies a differential equation of type (3.1) having property II. The corresponding function $f(x)$ is easily found. In the following example the graph $\Gamma$ of $y(x)$ is made up of arcs of parabolas of higher order and straight line segments fitted together so that $y(x)$ and $y^{\prime}(x)$ are continuous. The function $f(x)$ can then be read off from formula (1.2). Varying the orders of the parabolas and the relative lengths of the arcs, we can modify the properties of $g(x)$ as desired. This is the general idea, the details follow.

We observe first that if the parabola $y=C(x-s)^{1 / n}$ goes through the point $\left(x_{0}, y_{0}\right)$ with the slope $p_{0}$, then $C^{n}=n y_{0}^{n-1} p_{0}, s=x_{0}-y_{0} /\left(n p_{0}\right)$. The curve $\Gamma$ consists of arcs $\Gamma_{n}$ and line segments $\lambda_{n}$ following each other in the order $\Gamma_{0}, \lambda_{1}, \Gamma_{1}, \cdots, \Gamma_{n-1}, \lambda_{n}, \Gamma_{n}, \cdots$ and $\lambda_{n}$ is tangent to $\Gamma_{n-1}$ and $\Gamma_{n}$. Here $\Gamma_{0}$ is an arc of $y=x^{1 / 2}$ starting at $x=0$ and ending at $x=a_{1}$ while $\Gamma_{n}$ is an arc of $y=C_{n}\left(x-s_{n}\right)^{1 /(n+2)}$ starting at $x=a_{n}+b_{n}$ and ending at $x=a_{n+1}$, where $a_{n}<a_{n}+b_{n}<a_{n+1}$ and the values of $a_{n}$ and $b_{n}$ will be chosen later. The line segment $\lambda_{n}$ belongs to the straight line

$$
y=C_{n-1}\left(a_{n}-s_{n-1}\right)^{-(n+1) /(n+2)}\left[a_{n}-s_{n-1}+\frac{1}{n+2}\left(x-a_{n}\right)\right] .
$$

The values of $C_{n}$ and $s_{n}$ are uniquely determined by those of $a_{n}$ and $b_{n}$; the value of $C_{n}$ is immaterial but we need to know that

$$
s_{n}=\sum_{k=1}^{n} \frac{k+1}{k+2} b_{k} \text {. }
$$

We have

$$
f(x)=\frac{n+1}{(n+2)^{2}}\left(x-s_{n}\right)^{-2}, \quad a_{n}+b_{n}<x<a_{n+1},
$$

and zero elsewhere.

We shall now specialize $a_{n}$ and $b_{n}$. We choose

$$
a_{n}=2^{n^{2}}, \quad b_{n}=2^{n^{2}+n}
$$

and find that

$$
s_{n}=\frac{n+1}{n+2} 2^{n^{2}+n}\left[1+O\left(2^{-2 n}\right)\right] .
$$


If $2^{n^{2}} \leqq x \leqq 2^{n^{2}}+2^{n^{2}+n}$, then

$$
\int_{x}^{\infty} f(t) d t=\frac{n+1}{n+2} 2^{-n^{2}-n}\left[1+O\left(2^{-n}\right)\right] .
$$

It follows that

$$
g\left(2^{n^{2}}\right)=O\left(2^{-n}\right), \quad g\left(2^{n^{2}+n}\right)=\frac{n+1}{n+2}\left[1+O\left(2^{-n}\right)\right] .
$$

Hence $g_{*}=0$ and $g^{*}=1$ as asserted.

Among the further properties of this particular function the following should be noted. For $2^{n^{2}}\left(1+2^{n}\right) \leqq x \leqq 2^{(n+1)^{2}}$ we have

$$
u(x)=\frac{x}{(n+2)\left(x-s_{n}\right)} .
$$

A simple computation shows that $u(x)$ decreases from

$$
1+O\left(2^{-n}\right) \text { to } \frac{1}{n+2}\left[1+O\left(2^{-n}\right)\right]
$$

in this interval. Hence $u_{*}=0$ and $u^{*}=1$ which are the extreme limits of indetermination of a function $u(x)$ belonging to a differential equation of property II. In view of the discussion in $\$ 5$ it is also worth noting that

$$
\liminf _{x \rightarrow \infty} x^{2} f(x)=0, \quad \limsup _{x \rightarrow \infty} x^{2} f(x)=\infty .
$$

This concludes the discussion of example A.

B. $g_{*}=0$ does not imply property II.

Construction. We define $y(x)$ for $x \geqq 0$ by its graph which is made up of cosine arcs and horizontal line segments chosen in the following manner.

$$
\begin{array}{rlrl}
y(x) & =1, & 0 & \leqq x \leqq 1, \\
y(x) & =(-1)^{n+1}, & 2^{n^{2}+1} & \leqq x \leqq 2^{(n+1)^{2}}, \\
y(x) & =(-1)^{n} \cos \left(2^{-n^{2}} \pi x\right), & 2^{n^{2}} \leqq x \leqq 2^{n^{2}+1} .
\end{array}
$$

Here

$$
f(x)=2^{-2 n^{2}} \pi^{2}, \quad 2^{n^{2}} \leqq x \leqq 2^{n^{2}+1},
$$

and zero elsewhere. Further

$$
g\left(2^{n^{2}+1}\right)=2^{-2 n} \pi^{2}\left[1+O\left(2^{-n}\right)\right]
$$

so that $g_{*}=0$. Since $y(x)$ has infinitely many zeros, the equation cannot have property II.

C. $g^{*}=\frac{1}{4}$ does not imply property II. 
Construction. We take

$$
y(x)=x^{1 / 2}\left[C_{1}(\log x)^{\rho}+C_{2}(\log x)^{1-\rho}\right]
$$

where $\rho$ and $1-\rho$ are the two roots of the quadratic $u^{2}-u+\gamma_{1}=0$ and $\gamma_{1}>\frac{1}{4}$. Here

$$
f(x)=\frac{1}{4 x^{2}}+\frac{\gamma_{1}}{(x \log x)^{2}},
$$

so that $g_{*}=g^{*}=\frac{1}{4}$. Since $\rho$ is complex, the solutions are oscillatory and the equation does not have property II.

5. Extensions of the theorem of Kneser. As observed in the Introduction, A. Kneser used the methods of Sturm and example (1.2) to derive conditions under which equation (3.1) has property II. With a slight extension, we can formulate his result as follows.

THEOREM 9. Let

$$
\lim \sup _{\inf } x^{2} f(x)=\left\{\begin{array}{l}
\gamma^{*} \\
\gamma_{*}
\end{array} .\right.
$$

The solutions of (3.1) are non-oscillatory for large $x$ if $\gamma^{*}<\frac{1}{4}$, oscillatory if $\gamma_{*}>\frac{1}{4}$ and no conclusion can be drawn if either $\gamma^{*}$ or $\gamma_{*}$ equals $\frac{1}{4}$.

The proof of the first two assertions follows familiar lines and may be omitted here. Example $C$ at the end of $\S 4$ is one in which $\gamma_{*}=\gamma^{*}=\frac{1}{4}$; the solutions are non-oscillatory if $\gamma_{1} \leqq \frac{1}{4}$ and oscillatory if $\gamma_{1}>\frac{1}{4}$.

Example C suggests a further extension of Kneser's theorem. Cf. Rieman-Weber [7, p. 61] for Theorems 10 and 11.

THEOREM 10. Let

$$
\lim \sup _{\inf }(x \log x)^{2}\left[f(x)-\frac{1}{4 x^{2}}\right]=\left\{\begin{array}{l}
\gamma_{1}^{*} \\
\gamma_{1 *}
\end{array}\right.
$$

The solutions of (3.1) are non-oscillatory for largex if $\gamma_{1}^{*}<\frac{1}{4}$, oscillatory if $\gamma_{1 *}>\frac{1}{4}$, and no conclusion can be drawn if either $\gamma_{1}^{*}$ or $\gamma_{1 *}$ equals $\frac{1}{4}$.

The theorem is proved by the usual methods of Sturm using example C as comparison. The limiting cases require further counter examples.

As a matter of fact, examples (1.2) and $\mathrm{C}$ are merely the first instances of an infinite sequence of critical comparison equations which form a kind of logarithmic scale. To simplify the formulas, let us introduce some condensed notation. We write

$$
L_{0}(x)=x, \quad L_{p}(x)=L_{p-1}(x) \log _{p} x, \quad p=1,2,3, \cdots,
$$

where 


$$
\log _{2} x=\log \log x, \quad \log _{p} x=\log \log _{p-1} x .
$$

Further we set

$$
S_{p}(x)=\sum_{k=0}^{p}\left[L_{k}(x)\right]^{-2},
$$

and define $e_{1}=e, e_{k}=\exp \left(e_{k-1}\right)$. Then the functions

$$
y(x)=\left[L_{p-1}(x)\right]^{1 / 2}\left[C_{1}\left(\log _{p} x\right)^{\rho}+C_{2}\left(\log _{p} x\right)^{1-\rho}\right],
$$

where $\rho$ and $1-\rho$ are the two roots of the quadratic equation $u^{2}-u+\gamma_{p}=0$, satisfy a differential equation of type (3.1) with

$$
f(x)=\frac{1}{4} S_{p-1}(x)+\gamma_{p}\left[L_{p}(x)\right]^{-2},
$$$$
x>e_{p-1}
$$

This observation leads to

THEOREM 11. Let

$$
\lim \sup _{\inf }\left[L_{p}(x)\right]^{2}\left\{f(x)-\frac{1}{4} S_{p-1}(x)\right\}=\left\{\begin{array}{l}
\gamma_{p}^{*} \\
\gamma_{p *}
\end{array}\right.
$$

The solutions of (3.1) are non-oscillatory for large $x$ if $\gamma_{p}^{*}<\frac{1}{4}$, oscillatory if $\gamma_{p *}>\frac{1}{4}$, and no conclusion can be drawn if either $\gamma_{p}^{*}$ or $\gamma_{p *}$ eguals $\frac{1}{4}$.

The proof follows the same lines as the preceding theorems. None of these theorems is particularly good because the limits involved are too much affected by irregularities in $f(x)$. Cf. formula (3.1). These irregularities are smoothed out, to some extent at least, by an integration process which leads to more powerful criteria. Thus, combining the ideas of Theorems 7 and 11, we get

THEOREM 12. Equation (3.1) has property II if

$$
g(x) \leqq \frac{1}{4} x \int_{x}^{\infty} S_{p}(t) d t
$$

for $x$ sufficiently large.

Corollary 1 of Theorem 7 is the special case $p=0$ of this theorem.

6. An extension to the complex domain. The use of the singular Riccati integral equation (3.9) is not restricted to real variables. It can also be used to prove non-oscillation theorems in the complex domain. The results obtainable in this manner, though of a somewhat special nature, appear to be basically different from those derived by the present writer in the early nineteen twenties. The following is a sample of what can be done.

THEOREM 13. Let $f(z)$ be holomorphic in a sector $S:-\theta_{1}<\arg z<\theta_{2}$ of the 
complex plane and suppose that

$$
g(z)=z \int_{z}^{\infty} f(t) d t
$$

is well defined in $S$ when the integral is taken along a line parallel to the real axis. Finally, suppose that $|g(z)| \leqq \gamma<\frac{1}{4}$ for $z \in S$. Then there exists a solution $w(z)$ of the differential equation

$$
w^{\prime \prime}+f(z) w=0
$$

which has no zeros in $S$.

Proof. It is understood that $0<\theta_{1}, \theta_{2}<\pi$. We choose $\alpha$ so that $\gamma \alpha=\frac{1}{4} \sin \alpha$ and denote by $S_{\alpha}$ the intersection of $S$ with the sector $0<|z|<\infty,|\arg z|<\alpha$. Using the method of successive approximations we then construct a solution of

$$
u(z)=z \int_{z}^{\infty} u^{2}(t) \frac{d t}{t^{2}}+g(z)
$$

for $z \in S_{\alpha}$. The path of integration is taken parallel to the real axis. We set

$$
u_{0}(z)=2 \gamma, \quad u_{n}(z)=z \int_{z}^{\infty} u_{n-1}^{2}(t) \frac{d t}{t^{2}}+g(z) .
$$

Suppose that $\max \left|u_{n}(z)\right|=B_{n}$ for $z \in S_{\alpha}$. Then

$$
B_{n} \leqq \frac{\alpha}{\sin \alpha} B_{n-1}^{2}+\gamma,
$$

where we have used the formula

$$
r \int_{0}^{\infty}\left|r e^{i \theta}+s\right|^{-2} d s=\frac{\theta}{\sin \theta} .
$$

In view of the value of $B_{0}$ and the definition of $\alpha$, we conclude from (6.4) that $B_{n} \leqq 2 \gamma$ for all $n$. Thus the functions $u_{n}(z)$ are holomorphic in $S_{\alpha}$ and uniformly bounded. If $z=x$ is real, a simple computation shows that

$$
\left|u_{n}(x)-u_{n-1}(x)\right| \leqq 4 \gamma\left|u_{n-1}(x)-u_{n-2}(x)\right| \text {. }
$$

Hence the sequence $\left\{u_{n}(z)\right\}$ converges on the positive real axis. By the theorem of Vitali, $\lim _{n \rightarrow \infty} u_{n}(z)=u(z)$ exists and is holomorphic everywhere in $S_{\alpha}$. Further $u(z)$ satisfies (6.3).

If $S_{\alpha}$ exhausts $S$, we are through. If not, the fact that $|u(z)|$ is bounded in $S_{\alpha}$ enables us to modify the path of integration in (6.3). Let $\theta_{0}$ be real, $\left|\theta_{0}\right|<\max \left(\alpha, \theta_{1}, \theta_{2}\right)$, and replace the path of integration $\arg (t-z)=0$ by $\arg (t-z)=\theta_{0}$. Consider the sector $S_{\alpha}\left(\theta_{0}\right)$ which is the intersection of $S$ with $\left|\arg z-\theta_{0}\right|<\alpha, 0<|z|<\infty$. In $S \cap S_{\alpha}\left(\theta_{0}\right)$ the function $u(z)$ satisfies both the 
original integral equation and the new one with the modified path. The convergence proof then shows that the same sequence of approximations converges to a holomorphic function in $S_{\alpha}\left(\theta_{0}\right)$. By a suitable repetition of this process, we can extend $u(z)$ analytically throughout $S$.

Once we have a solution $u(z)$ of (6.3) which is holomorphic in $S$, we see that $v(z)=u(z) / z$ satisfies the Riccati equation $v^{\prime}+v^{2}+f(z)=0$ everywhere in $S$, and placing $w(z)=\exp \left[\int_{1}^{z} v(t) d t\right]$ we have a solution of (6.2) which is different from zero everywhere in $S$. This completes the proof.

It is natural to ask if the value zero is of low frequency in $S$ for every solution of (6.2). This is certainly true in simple cases; whether or not it is generally true requires further investigation.

\section{BIBLIOGRAPHY}

1. R. Bellman, The boundedness of solutions of linear differential equations, Duke Math. J. vol. 14 (1947) 83-97.

2. E. Cotton, Sur les solutions asymptotiques des équations différentielles, Ann. Ecole Norm. (3) vol. 28 (1911) pp. 473-521.

3. W. B. Fite, Concerning the zeros of the solutions of certain differential equations, Trans. Amer. Math. Soc. vol. 19 (1917) pp. 341-352. $488-493$.

4. E. Hille, A general type of singular point, Proc. Nat. Acad. Sci. U.S.A. vol. 10 (1924) pp.

5. A. Kneser, Untersuchungen über die reellen Nullstellen der Integrale linearer Differentialgleichungen, Math. Ann vol. 42 (1893) pp. 409-435.

6. N. Levinson, The growth of the solutions of a differential equation, Duke Math. J. vol. 8 (1941) pp. 1-10.

7. B. Riemann-H. Weber, Die partiel'en Differentialgleichungen der Mathematischen Physik, vol. II, 5th ed., Braunschweig, 1912, xiv +575 pp.

8. G. N. Watson, A treatise on the theory of Bessel functions, Camridge, 1944, viii $+804 \mathrm{pp}$.

9. A. Wiman, Über die reellen Lösungen der linearen Differentialgleichungen zweiter Ordnung, Arkiv för Matematik, Astronomi och Fysik vol. 12, no. 14 (1917) 22 pp.

10. A. Wintner, On the Laplace-Fourier transcendents occurring in mathematical physics, Amer. J. Math. vol. 69 (1947) pp. 87-98.

YALE UNIVERSITY,

New Haven, Cons. 